\title{
A PROBLEM IN THE PROPAGATION OF SHOCK*
}

\author{
BY \\ MONROE H. MARTIN \\ University of Maryland
}

Introduction. This paper deals with a single problem in the rectilinear motion of a gas, namely, what is the subsequent behavior of a gas initially at rest if its initial density is a constant $\rho_{0}$ in the region $|x|<1$ and a constant $\rho_{2}<\rho_{0}$ in the region $|x|>1$ ?

The behavior of the gas is an idealization of the behavior of the atmosphere in an infinitely long right circular cylinder after an explosion within the cylinder.

It is assumed that the pressure $p$ and density $\rho$ of the gas are related by the isentropic law $p=k^{2} \rho^{\gamma}$ where $k^{2}$ is constant for all $x$ and all $t$. Under the law of conservation of energy ${ }^{1}$ (Rankine-Hugoniot equation) there is a change ${ }^{2}$ in entropy across a shock and the results in the paper may be regarded as an approximation to the actual state of affairs only in the case where the change in density across the shock is very small with a correspondingly small change in entropy.

At times the author has not hesitated to restrict attention to a monatomic gas $(\gamma=5 / 3)$ in order to avoid formal mathematical difficulties. ${ }^{3}$ The behavior of the gas undergoes marked changes as the difference $\rho_{0}-\rho_{2}$ between the initial densities is permitted to vary. ${ }^{4}$

If $\rho_{0}-\rho_{2}$ is sufficiently small the two initial shocks give rise to shocks traveling in opposite directions towards infinity as $t$ increases indefinitely. Up to a certain instant the shocks travel with constant velocity greater than the velocity of sound in the undisturbed gas. After this instant their velocity of propagation decreases monotonically with time, to approach the velocity of sound in the undisturbed gas as the shocks recede to infinity. ${ }^{5}$ The behavior of the gas between the two shocks is followed up to a stage when the mapping ${ }^{6}$ of Riemann's $(r, s)$-plane upon the $(x, t)$-plane loses its oneto-one character. The further behavior of the gas still awaits determination.

Plates 1 and 2 at the end of the paper present qualitatively the variation of density (or pressure), over the gas for $\rho_{0}-\rho_{2}$ sufficiently small.

1. Fundamental principles. Assuming that the pressure $p$ is a monotonic increasing function of the density $\rho$ and denoting the velocity by $u$, the partial differential equations

* Received March 8, 1946.

${ }^{1}$ See Riemann-Weber, Die partiellen Differentialgleichungen der Mathematischen Physik, 6th ed., Friedr. Vieweg \& Sohn Brawnschweig, 1919, vol. 2, pp. 549-550.

${ }^{2}$ Indeed under the Rankine-Hugoniot hypothesis it follows from formula (10) on p. 513 of RiemannWeber, op. cit. that the entropy of the gas in back of the shock depends upon the ratio of the densities of the gas on the two sides of the shock. This ratio changes as the shock propagates and consequently the the entropy of the gas in back of the shock is not constant.

${ }^{3}$ The examination of other values of $\gamma$ has been begun by R. C. Rand in his doctorate thesis entitled The rectilinear motion of a gas subsequent to an internal explosion. A copy of this thesis is on file in the library of the University of Maryland.

${ }^{4}$ R. C. Rand, loc. cit.

- See, however, the last sentence in $\$ 6$ of the present paper.

6 For a discussion of this mapping see Riemann-Weber, loc. cit., pp. 533-536 or The rectilinear motion of a gas, Amer. J. Math. 65, 391-401 (1943). This paper will be cited as I. 


$$
\rho\left(u_{t}+u_{x} u\right)+G^{2} \rho_{x}=0, \quad \rho_{t}+(\rho u)_{x}=0, . G^{2}=G^{2}(\rho)=p^{\prime},
$$

for $u, \rho$ become

$$
r_{t}+\alpha r_{x}=0, \quad s_{t}+\beta s_{x}=0, \quad \alpha=u+G, \quad \beta=u-G,
$$

if we set

$$
u=r+s, \quad \int_{0}^{\rho}(G / \rho) d \rho=r-s>0 .
$$

Clearly $u, \rho$ are monotonic increasing functions of $r+s, r-s$ respectively and

$$
\alpha=\alpha(r, s)=r+s+G(\rho(r-s)), \quad \beta=\beta(r, s)=r+s-G(\rho(r-s))
$$

satisfy

$$
\alpha(-s,-r)=-\beta(r, s), \quad \beta(-s,-r)=-\alpha(r, s) .
$$

A point of the $(u, \rho)$-plane, or its correspondent by $(2)$ in the $(r, s)$-plane, is said to represent or be a state of the gas. The points of the $(r, s)$-plane representing states of the gas comprise a half-plane $r \geqq s$ termed the state plane. Points representing states having the same velocity (density) lie on the lines $r+s=$ const. $(r-s=$ const.) and the velocity (density) of a state increases with the distance of the point $(r, s)$ from the line of zero velocity $r=-s$ (the line of zero density $r=s$ ). The velocity is positive or negative according as $(r, s)$ lies above or below the line $r=-s$.

In general a solution $r=r(x, t), s=s(x, t)$ of (1) transforms a region of the $(x, t)$ plane into a region in the state plane and is single valued. The inverse transformation $T: x=x(r, s), t=t(r, s)$ is not necessarily single-valued and is regarded as assigning the state $(r, s)$ to its transform $(x, t)$. Corresponding to (1) there is the system

$$
x_{r}-\beta t_{r}=0, \quad x_{s}-\alpha t_{s}=0,
$$

of partial differential equations for $x(r, s), t(r, s)$ in $T$. The Jacobian $J$ of $T$ is

$$
J=-(\alpha-\beta) t_{r} t_{s}=-2 G t_{r} t_{s} .
$$

If $x, t$ are solutions of (5), the system of Pfaff

$$
d w=(x-\alpha t) d r+(x-\beta t) d s, \quad d v=2 \frac{x-\alpha t-v}{\alpha-\beta} d r-2 \frac{x-\beta t-v}{\alpha-\beta} d s,
$$

is completely integrable, and conversely. When we write

$$
x-\alpha t=w_{r}, \quad x-\beta t=w_{s},
$$

the integrability condition for the second equation becomes ${ }^{7}$

$$
(\alpha-\beta) w_{r s}-\beta_{r}\left(w_{r}-w_{s}\right)=0 .
$$

Taking $w$ a solution of (8) it follows from (7) that a transformation $T$ is

$$
T_{w}: \quad x=-\frac{\beta w_{r}-\alpha w_{s}}{\alpha-\beta}, \quad t=-\frac{w_{r}-w_{s}}{\alpha-\beta} .
$$

The following theorem is a direct consequence of (4).

${ }^{7}$ Cf. Riemann-Weber, loc. cit., pp. 536-538 or pp. 393-394 of I. 
THEOREM 1. Given $w=w(r, s)$ a solution of (8), another solution is $\bar{w}=w(-s,-r)$ and $T_{w}, T_{\bar{w}}$ map points which are reflections of each other in the line of zero velocity $r=-s$, into points which are reflections of each other in the line $x=0$.

As a corollary, we see that if $w(r, s)=w(-s,-r)$ points which are reflections of each other in the line $r=-s$, are carried by $T_{w}$ into points which are reflections of each other in $x=0$.

The theorem is obvious $a$ priori on physical grounds. Given any motion of the gas, its particles may be reflected in the plane $x=0$ to gain another motion.

Taking $r=r_{0}=$ const., the second equation in (1) upon multiplication by $d \beta / d s$ becomes

$$
\beta_{t}+\beta \beta_{x}=\frac{\partial(x-\beta t, \beta)}{\partial(x, t)}=0,
$$

and therefore a solution of (1) is given implicitly by ${ }^{8}$

$$
r=r_{0}, \quad x-\beta t=\Psi(\beta),
$$

$\Psi(\beta)$ denoting an arbitrary function of $\beta$. Corresponding to $s=s_{0}=$ const., a solution of (1) is obtained from

$$
x-\alpha t=\Phi(\alpha), \quad s=s_{0} .
$$

For a fixed $s$ in $(9)$ the state $\left(r_{0}, s\right)$ is assigned ${ }^{9}$ to all points of the straight line $x-\beta t=\Psi(\beta)$. This line is termed a propagation line and the state $\left(r_{0}, s\right)$ is said to be propagated along it. Physically the state $\left(r_{0}, s\right)$ is propagated through the gas with a velocity $\beta$ with respect to a fixed plane.

Let us assume that $T_{w}$ puts the states of a region $R$ of the state plane in (one-toone correspondence with the points of a region $X$ of the $(x, t)$-plane. The transform by $T_{w}$ of a segment of $r=$ const. ( $s=$ const.) in $R$ is a curve in $X$ termed an $r$-curve (s-curve). The $r$ and $s$-curves provide a curvilinear coordinate system on $X$ from which the state of the gas may be read off at any point of $X$.

From (5) the slope of an $r(s)$-curve ${ }^{10}$ is $1 / \alpha(1 / \beta)$; from (9), (10) the propagation lines drawn from the points of an $r(s)$-curve have slope $1 / \beta(1 / \alpha)$. Therefore the tangents drawn to $s(r)$-curves at the points of an $r(s)$-curve are propagation lines and, in so far as they do not intersect, may be used to assigned the states on the $r(s)$-curve to the points of the region covered by them.

Two $r(s)$-curves $C, \bar{C}$ transforms of $r=r_{0}\left(s=s_{0}\right)$ under $T_{w}, T_{\bar{w} w}$ respectively are said to be propagated from each other if the propagation lines drawn from points of $C, \bar{C}$ which are transforms of the same state are identical.

Lemma 1. Two $r[s]$-curves $C, \bar{C}$ transforms by $T_{w}, T_{\bar{w}}$ of $r=r_{0}\left[s=s_{0}\right]$ are propagated from each other if, and only if $w_{s}\left(r_{0}, s\right)=\bar{w}_{s}\left(r_{0}, s\right)\left[w_{r}\left(r, s_{0}\right)=\bar{w}_{r}\left(r, s_{0}\right)\right]$.

From (7) parametric equations of $C, \bar{C}$ are

$$
\begin{array}{lll}
C: & x-\alpha\left(r_{0}, s\right) t=w_{r}\left(r_{0}, s\right), & x-\beta\left(r_{0}, s\right) t=w_{s}\left(r_{0}, s\right), \\
\bar{C}: \quad & x-\alpha\left(r_{0}, s\right) t=\bar{w}_{r}\left(r_{0}, s\right), & x-\beta\left(r_{0}, s\right) t=\bar{w}_{s}\left(r_{0}, s\right) .
\end{array}
$$

${ }^{8}$ Cf. Riemann-Weber, loc. cit., p. 518.

${ }^{\circ}$ Cf. Riemann-Weber, loc. cit., pp. 516-520.

${ }^{10}$ First noted by R. C. Rand. 
Along a propagation line propagating the state $\left(r_{0}, s\right)$ we have $x-\beta\left(r_{0}, s\right) t=$ const. Hence propagation lines drawn from a point on $C$ and a point on $\bar{C}$, both transforms of the same state $\left(r_{0}, s\right)$ will be identical if, and only if, $w_{s}\left(r_{0}, s\right)=\bar{w}_{s}\left(r_{0}, s\right)$.

It is interesting to note that tangents drawn to $C, \bar{C}$ at points which are transforms of the same state are parallel.

Lemma 2. Given two $r[s]$-curves $C, \bar{C}$ which are propagated from each other, curve $\bar{C}$ will pass through a point $(\bar{x}, \bar{t})$ on the propagation line propagating the state $\left(r_{0}, \bar{s}\right)$, $\left[\left(\bar{r}, s_{0}\right)\right]$ from $C$ if and only if $\bar{w}_{r}\left(r_{0}, \bar{s}\right)=\bar{x}-\alpha\left(r_{0}, \bar{s}\right) \bar{i}\left[\bar{w}_{s}\left(\bar{r}, s_{0}\right)=\bar{x}-\beta\left(\bar{r}, s_{0}\right) \bar{t}\right]$. This condition determines $\bar{w}_{r}\left(r_{0}, s\right)\left[\bar{w}_{s}\left(r, s_{0}\right)\right]$ uniquely.

The first part of the lemma follows from the parametric equations of $\bar{C}$. To prove the second part we set $r=r_{0}, w=\bar{w}$ in (8) to obtain an ordinary, linear differential equation for $\bar{w}_{r}$ since $\bar{w}_{s}=w_{s}$ is a known function of $s$. This determines $\bar{w}_{r}$ uniquely, for $\bar{w}_{r}$ is known when $s=\bar{s}$.

2. Shocks and buffer waves. Under the assumption that $G$ increases with $\rho$ it follows that $G=G(\rho(r-s))$ is an increasing [decreasing] function of $r[s]$ for fixed $s[r]$; from (3), one concludes that $\alpha[\beta]$ is an increasing function of $r[s]$ for fixed $s[r]$.

Lemma 3. If initially $r=r_{0}$ for $-\infty<x<+\infty$ and $s=s_{1}$ or $s_{2}$ as $x<0$ or $x>0$ with $s_{1}<s_{2}$, subsequently the state of the gas is unchanged exterior to the "buffer region" between the lines $x=\beta\left(r_{0}, s_{1}\right) t, x=\beta\left(r_{0}, s_{2}\right) t$. Within this region the state $\left(r_{0}, s\right)$ with $s_{1}<s<s_{2}$ is propagated $^{11}$ along the propagation line $x=\beta\left(r_{0}, s\right)$.

Initial states are propagated along the propagation lines

$$
x-\beta\left(r_{0}, s_{1}\right) t=k_{1}<0, \quad x-\beta\left(r_{0}, s_{2}\right) t=k_{2}>0, \quad \beta\left(r_{0}, s_{1}\right)<\beta\left(r_{0}, s_{2}\right),
$$

which diverge as shown in Figure 1 to assign the state $\left(r_{0}, s_{1}\right)$ to the region on the left of $O A_{1}$ and the state $\left(r_{0}, s_{2}\right)$ to the region on the right of $O A_{2}$. To obtain the states in

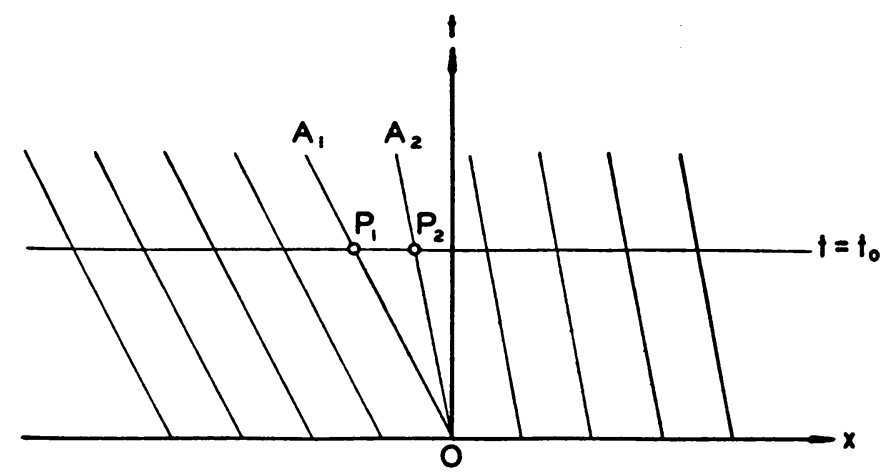

Fig. 1.

the buffer region $A_{1} O A_{2}$ one sets $\Psi(\beta) \equiv 0$ in (9) and draws the propagation line $x=\beta\left(r_{0}, s\right) t$ from 0 . Along this propagation line the state is $\left(r_{0}, s\right)$ and as $s$ ranges from $s_{1}$ to $s_{2}$ the propagation line turns from $O A_{1}$ to $O A_{2}$ to assign states to all points of the 
buffer region. It will be observed that the states vary continuously along a line $t=t_{0}>0$.

In this solution of (1), the inverse transformation $T$ is not single-valued the, segment $s_{1} \leqq s \leqq s_{2}$ of $r=r_{0}$ being carried by $T$ into the half-plane $t \geqq 0$.

Physically the buffer region corresponds to a disturbance $P_{1} P_{2}$ affecting two bodies of gas of different uniform states in contact with one another initially, the end points of the disturbance traveling with the local velocity of sound in the two bodies of gas. The passage of this disturbance through the gas is termed a buffer wave.

$A$ shock exists at $x=\xi$ if $\rho_{1} \neq \rho_{2}$ and is propagated with a velocity ${ }^{12}$

$$
\dot{\xi}=u_{1} \pm \sqrt{\frac{\rho_{2}}{\rho_{1}} \frac{p_{1}-p_{2}}{\rho_{1}-\rho_{2}}}=u_{2} \pm \sqrt{\frac{\rho_{1}}{\rho_{2}} \frac{p_{1}-p_{2}}{\rho_{1}-\rho_{2}}}
$$

where

$$
\begin{array}{lll}
u_{1}=u(\xi-0), & \rho_{1}=\rho(\xi-0), & p_{1}=p(\xi-0), \\
u_{2}=u(\xi+0), & \rho_{2}=\rho(\xi+0), & p_{2}=p(\xi+0) .
\end{array}
$$

The curve $x=\xi(t)$ in the $(x, t)$-plane is termed a shock curve. It will be sufficient for the purposes of this investigation to consider progressive condensation shocks arising when $\rho_{1}>\rho_{2}$ and the positive sign is taken in (11). For a shock of this type one has the condition

$$
u_{1}-u_{2}=\sqrt{\left(p_{1}-p_{2}\right)\left(\rho_{2}^{-1}-\rho_{1}^{-1}\right)}
$$

with

$$
\xi=\left(u_{1} \rho_{1}-u_{2} \rho_{2}\right)\left(\rho_{1}-\rho_{2}\right)^{-1} .
$$

If $\left(r_{1}, s_{1}\right),\left(r_{2}, s_{2}\right)$ denote the correspondents of $\left(u_{1}, \rho_{1}\right),\left(u_{2}, \rho_{2}\right)$ by $(2)$ and the state $\left(r_{2}, s_{2}\right)$ on the right of the shock is given, the state $\left(r_{1}, s_{1}\right)$ on the left of the shock is not uniquely determined but, by (12), may be any point of the curve.

$$
r+s=r_{2}+s_{2}+\sqrt{\left(p-p_{2}\right)\left(\rho_{2}^{-1}-\rho^{-1}\right)}, \quad p=p(r-s), \quad \rho=\rho(r-s)>\rho_{2}
$$

in the state plane. This curve is termed the compatibility curve of the state $\left(r_{2}, s_{2}\right)$ and its equation may be written in the parametric form

$$
\begin{aligned}
& r=\frac{1}{2}\left\{r_{2}+s_{2}+v+\sqrt{\left(p-p_{2}\right)\left(\rho_{2}^{-1}-\rho^{-1}\right)}\right\}, \\
& s=\frac{1}{2}\left\{r_{2}+s_{2}-v+\sqrt{\left(p-p_{2}\right)\left(\rho_{2}^{-1}-\rho^{-1}\right)}\right\}, \quad v>v_{2},
\end{aligned}
$$

upon introducing the parameter $v=r-s$, where, of course, $v_{2}=r_{2}-s_{2}$.

LemMa 4. The compatibility curve of a state $\left(r_{2}, s_{2}\right)$ rises with increasing $r$ from the point $\left(r_{2}, s_{2}\right)$, at which it has a horizontal tangent.

Both derivatives of $r, s$ with respect to $v$ will be positive provided

$$
\frac{\rho}{\rho_{2}} G^{2}(\rho)>\frac{p-p_{2}}{\rho-\rho_{2}}=G^{2}(\bar{\rho}) \text { where } \rho_{2}<\bar{\rho}<\rho,
$$

12 See, for example, Riemann-Weber, op. cit. p. 513. 
which inequality holds for $\rho>\rho_{2}$ under the assumption that $G$ increases with $\rho$.

We shall now consider the simultaneous generation of a shock and buffer wave ${ }^{13}$ as pictured in Figure 2 where $B$ is a buffer region between two regions $R_{0}, R_{1}$ of uniform state $\left(r_{0}, s_{0}\right),\left(r_{1}, s_{1}\right)$ respectively and $O S$ is the shock line separating $R_{1}$ from the region $R_{2}$ of uniform state $\left(r_{2}, s_{2}\right)$.

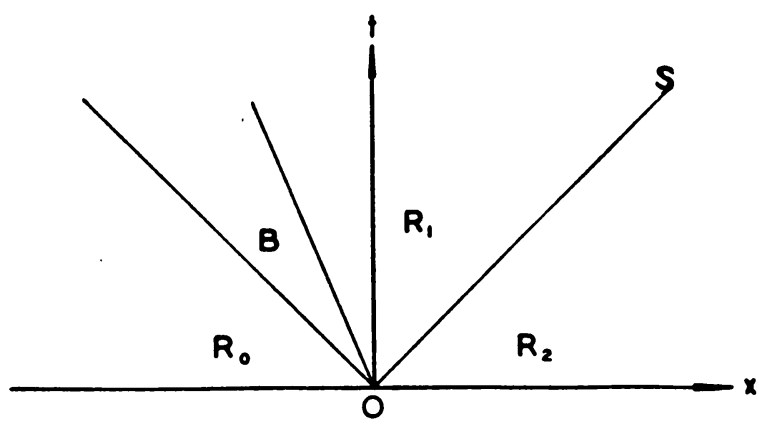

Fig. 2.

LEMMA 5. A shock and buffer wave are generated simultaneously ${ }^{13}$ at the contact of two bodies of gas of different uniform states $\left(r_{0}, s_{0}\right),\left(r_{2}, s_{2}\right)$ provided the point $\left(r_{0}, s_{0}\right)$ in the state plane lies directly underneath the compatibility curve of the state $\left(r_{2}, s_{2}\right)$.

Choosing the state $\left(r_{2}, s_{2}\right)$ in $R_{2}$ arbitrarily, the state in $R_{1}$ must be represented by a point on the compatibility curve of the state $\left(r_{2}, s_{2}\right)$; and if this point lies directly above $\left(r_{0}, s_{0}\right)$ the existence of the buffer region $B$ is assured by Lemma 3 .

3. The isentropic case. Here $p=k^{2} \rho^{\gamma}$ with $k, \gamma>1$ constants and $G$ increases with $\rho$ so that the results of $\$ 2$ remain in force. Moreover

$$
G=\frac{\gamma-1}{2}(r-s), \quad \alpha=\frac{1+\gamma}{2} r+\frac{3-\gamma}{2} s, \quad \beta=\frac{3-\gamma}{2} r+\frac{1+\gamma}{2} s,
$$

and (8) becomes

$$
(r-s) w_{r s}-m\left(w_{r}-w_{s}\right)=0, \quad m=\frac{3-\gamma}{2(\gamma-1)} .
$$

For monatomic gases $\gamma=5 / 3$ one finds $\alpha=\frac{2}{3}(2 r+s), \beta=\frac{2}{3}(r+2 s)$. Also $m=1$ and (16) becomes

$$
(r-s) w_{r s}-\left(w_{r}-w_{s}\right)=0,
$$

the general solution of which is

$$
w=\frac{R-S}{r-s}, \quad R=R(r), \quad S=S(s),
$$

$R(r), S(s)$ being arbitrary functions. The transformation $T_{w}$ is

or, from (17)

$$
x=-\frac{(r+2 s) w_{r}-(2 r+s) w_{s}}{r-s}, \quad t=-\frac{3}{2} \frac{w_{r}-w_{s}}{r-s},
$$

$$
\begin{aligned}
& x=3 \frac{r+s}{(r-s)^{3}}(R-S) \div \frac{(r+2 s) R^{\prime}+(2 r+s) S^{\prime}}{(r-s)^{2}}, \\
& t=\frac{3(R-S)}{(r-s)^{3}}-\frac{3}{2} \frac{R^{\prime}+\frac{S^{\prime}}{(r-s)^{2}},}{}
\end{aligned}
$$

${ }^{13}$ Cf. Riemann-Weber, loc. cit., pp. 527-529. 
so that

$$
\begin{aligned}
& t_{r}=-\frac{3}{2} \frac{(r-s)^{2} R^{\prime \prime}-2(r-s)\left(2 R^{\prime}+S^{\prime}\right)+6(R-S)}{(r-s)^{4}}, \\
& t_{s}=-\frac{3}{2} \frac{(r-s)^{2} S^{\prime \prime}+2(r-s)\left(R^{\prime}+2 S^{\prime}\right)-6(R-S)}{(r-s)^{4}},
\end{aligned}
$$

primes denoting differentiations of $R, S$ with respect to their arguments.

LEMma 6. For a monatomic gas the compatibility curve of a state $\left(r_{2}, s_{2}\right)$ is an arc of an algebraic curve of eighth degree ending at $\left(r_{2}, s_{2}\right)$, about which the points of the arc permit the expansions

$r=r_{2}+v-v_{2}+\kappa\left(v-v_{2}\right)^{3}+\cdots, \quad s=s_{2}+\kappa\left(v-v_{2}\right)^{3}+\cdots, \quad k=1 / 3 v_{2}^{2}$.

At all other points $r$ is a regular analytic function of $s$ with a positive derivative and $r, s$ are regular analytic functions (14') of the uniformizing parameter $v$, with respect to which they possess positive derivatives.

For monatomic gases equations (14), (14') become

$$
\begin{aligned}
r+s & =r_{2}+s_{2}+\sqrt{\frac{1}{16}}\left[\overline{(r-s)^{5}-\left(r_{2}-s_{2}\right)^{5}}\right]\left[\overline{\left(r_{2}-s_{2}\right)^{-3}-(r-s)^{-3}}\right], \\
r & =\frac{1}{2}\left[r_{2}+s_{2}+v+\sqrt{\frac{1}{13}\left(v^{5}-v_{2}^{5}\right)\left(v_{2}^{-3}-v^{-3}\right)}\right], \\
s & =\frac{1}{2}\left(r_{2}+s_{2}-v+\sqrt{\frac{1}{15}\left(v^{5}-v_{2}\right)\left(v_{2}^{-8}-v^{-3}\right)}\right],
\end{aligned}
$$

from which the statements in the lemma follow straight forwardly.

We return to the general adiabatic case. A point in the state plane represents a state for which the velocity is subsonic, sonic or supersonic according as the point lies in, on the boundary of, or exterior to the region. $\alpha>0, \beta<0$ between the straight lines $\alpha=0, \beta=0$.

LEMMA 7. The angle of inclination $\theta$ of the tangent at a point of an $r[s]$-curve is less [greater] than the angle of inclination $\phi$ of the propagation line drawn from this point. Both angles lie between 0 and $\pi$ and are decreasing functions of $s[r]$.

The lemma is obvious in view of (15) and previous results in $\$ 1$ on the slopes of $r, s$-curves and propagation lines.

LEMMA 8. If $T_{w}$ puts a region $R$ of the state plane in (1-1) correspondence with a region $X$ of the $(x, t)$-plane and if the Jacobian $J$ of $T_{w}$ never vanishes in $R$, the curvature of an $r[s]$-curve in $X$ has a fixed sign and the parts of the propagation lines drawn on the convex side do not intersect.

4. The first initial value problem. Returning to the problem formulated in the introduction, the correspondents of the initial states $\left(0, \rho_{0}\right),\left(0, \rho_{2}\right)$ of the gas are represented by the points $P_{0}\left(r_{0}, s_{0}\right), P_{2}\left(r_{2}, s_{2}\right)$ of the state plane in Figure 3a. Both $P_{0}, P_{2}$ lie on the line of zero velocity $r+s=0$, with $r_{0}>r_{2}$, since $\rho_{0}>\rho_{2}$.

From Lemma 4 we observe that $P_{0}$ lies directly underneath a point $Q\left(r_{0}, s_{1}\right)$ of the compatibility curve of $P_{2}$ and therefore, according to Lemma 5, a shock and buffer wave are generated simultaneously in the gas at $x=1$. In Figure $3 \mathrm{~b}$ the shock 
line from $A(1,0)$ is $A Q^{\prime \prime}$ and the buffer region is $P_{0}^{\prime} A Q^{\prime}$. States in the regions $O A P_{0}^{\prime}, Q^{\prime} A Q^{\prime \prime}, Q^{\prime \prime} A x$ are represented by points $P_{0}, Q, P_{2}$ respectively in Figure $3 a$. It is obvious from symmetry considerations that a shock line $\bar{A} \bar{Q}^{\prime \prime}$ and a buffer region

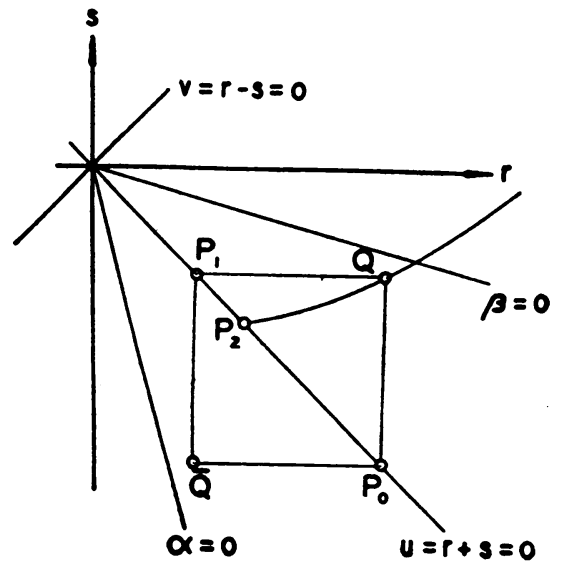

FIG. 3a.

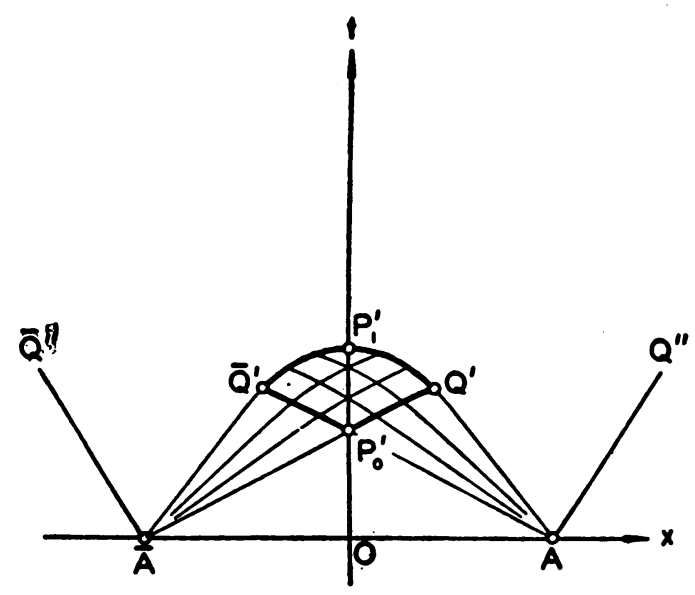

Fig. 3b.

$P_{0}^{\prime} \bar{A} \bar{Q}^{\prime}$ emanate from $\bar{A}(-1,0)$ and that states in the regions $O \bar{A} P_{0}^{\prime}, \bar{Q}^{\prime} \bar{A} \bar{Q}^{\prime \prime}, \bar{Q}^{\prime \prime} \bar{A} x$ are represented by points $P_{0}, \bar{Q}, P_{2}$ in Figure 3 a with $\bar{Q}$ the reflection of $Q$ in the line $r+s=0$. In the buffer region emanating from $A[\bar{A}]$, we have $r=r_{0}\left[s=s_{0}\right]$ and the equations of the propagation line are

$$
x-\beta\left(r_{0}, s\right) t=+1, \quad s_{0} \leqq s \leqq s_{1}, \quad\left[x-\alpha\left(r, s_{0}\right) t=-1, \quad \begin{array}{c}
\left.r_{1} \leqq r \leqq r_{0}\right] \\
\left(r_{1}=-s_{1}\right) .
\end{array}\right.
$$

As $s[r]$ ranges from $s_{0}\left[r_{0}\right]$ to $s_{1}\left[r_{1}\right]$ the propagation line from $A[\bar{A}]$ turns from $A P_{0}^{\prime}\left[\bar{A} P_{0}^{\prime}\right]$ to $A Q^{\prime}\left[\bar{A} \bar{Q}^{\prime}\right]$, with $t=G_{0}^{-1}$ where $G_{0}=G\left(\rho_{0}\right)$ at $P_{0}^{\prime}$. These propagation lines intersect on the $t$-axis above $P_{0}^{\prime}$ to assign different states to their intersection points. We avoid such a physical impossibility by terminating them on the arcs $P_{0}^{\prime} Q^{\prime}, P_{0}^{\prime} \bar{Q}^{\prime}$ in Figure $3 \mathrm{~b}$. The propagation lines assign the states on $\bar{Q} P_{0} Q$ to the points of $\bar{Q}^{\prime} P_{0}^{\prime} Q^{\prime}$ and we seek a $T_{w}$ which carries $\bar{Q} P_{0} Q$ into $\bar{Q}^{\prime} P_{0}^{\prime} Q^{\prime}$ and assigns the same states to the same points of the latter arc. A comparison of (7) with (22) leads to the following initial value problem.

The First Initial Value Problem. Given two constants $r_{0}$, $s_{0}$, find a solution $w^{(1)}$ of (16) for which $w_{r}^{(1)}\left(r, s_{0}\right)=-1, w_{s}^{(1)}\left(r_{0}, s\right)=+1$.

Before giving the solution for the general adiabatic case, we recall a few facts concerning the resolvent ${ }^{14}$ of (16). This resolvent is a two parameter family of solutions $v=v\left(r, s ; r_{0}, s_{0}\right)$ of the conjugate equation $(r-s) v_{r s}+m\left(v_{r}-v_{s}\right)=0$ meeting the initial conditions $v_{r}\left(r, s_{0} ; r_{0}, s_{0}\right)=+1, v_{s}\left(r_{0}, s ; r_{0}, s_{0}\right)=-1$, and is given by

$$
\begin{aligned}
v= & \left(r-r_{0}\right)\left(\frac{r_{0}-s}{r_{0}-s_{0}}\right)^{m} F_{1}\left(1-m ; m ;-m ; 2 ; \frac{r-r_{0}}{s_{0}-r_{0}} ; \frac{r-r_{0}}{s-r_{0}}\right) \\
& -\left(s-s_{0}\right)\left(\frac{r-s_{0}}{r_{0}-s_{0}}\right)^{m} F_{1}\left(1-m ; m ;-m ; 2 ; \frac{s-s_{0}}{r_{0}-s_{0}} ; \frac{s-s_{0}}{r-s_{0}}\right),
\end{aligned}
$$

${ }^{14}$ See $I$, in particular $\$ 3$ and $\$ 5$. 
where $F_{1}$ is Appell's first hypergeometric function of two variables. The solution $w^{(1)}$ of the first initial value problem is obtained by replacing $m$ by $-m$ and changing the sign of the resolvent.

Monatomic gases present the simplest mathematical problem and from now on they will receive our attention exclusively. For them $m=1$ and

$$
w^{(1)}=-\frac{\left(r-r_{0}\right)\left(r-s_{0}\right)+\left(s-r_{0}\right)\left(s-s_{0}\right)}{r-s}=\frac{r_{0}^{2}-r^{2}-\left(s^{2}-s_{0}^{2}\right)}{r-s},
$$

the last equation holding provided $r_{0}+s_{0}=0$.

Comparison of (17) and (23) yields

$$
R=r_{0}^{2}-r^{2}, \quad S=s^{2}-s_{0}^{2},
$$

so that $\left(18^{\prime}\right)$ and $(19)$ become

$$
\begin{array}{ll}
x^{(1)}=-(r+s) \frac{(r-s)^{2}+6\left(r s+r_{0} s_{0}\right)}{(r-s)^{2}}, & t^{(1)}=-6 \frac{r s+r_{0} s_{0}}{(r-s)^{3}}, \\
t_{r}^{(1)}=9(r-s)^{-4}\left(\alpha s-2 r_{0}^{2}\right) & t_{0}^{(1)}=-9(r-s)^{-4}\left(\beta r-2 r_{0}^{2}\right),
\end{array}
$$

where the superscripts record that $w=w^{(1)}$ in $T_{w}$. In the subsonic region of the state plane $\alpha>0, \beta<0$ and, therefore in this region

$$
i_{r}^{(1)}<0, \quad t_{s}^{(1)}>0, \quad J^{(1)}>0 .
$$

The square $P_{0} Q P_{1} \bar{Q}$ in Figure $3 \mathrm{a}$ is termed the primary region. As $\rho_{0}$ increases from $\rho_{2}$ the primary region expands from point $P_{2}$ till eventually $P_{1}$ leaves the state plane. We consider only values of $\rho_{0}$ for which the primary region lies entirely in the subsonic region and forego examination of the several interesting cases which arise $\mathrm{e}^{15}$ when this is not the case.

Arc $P_{0}^{\prime} Q^{\prime}$, the transform of $P_{0} Q$ by $T_{w}(1)$, is tangent to $\bar{A} P_{0}^{\prime}$ at $P_{0}^{\prime}$ and has slope $\tan \theta=1 / \alpha>0$. Since $t^{(1)}$ increases by (27) and the acute angle $\theta$ decreases by Lemma 7 with increasing $s$, arc $P_{0}^{\prime} Q^{\prime}$ is concave downwards. Likewise arc $Q^{\prime} P_{1}^{\prime}$ the transform of $Q P_{1}$ by $T_{w}^{(1)}$, is concave downwards. From (23) and the corollary to Theorem 1 it follows that arcs $P_{0}^{\prime} \bar{Q}^{\prime}, \bar{Q}^{\prime} P_{1}^{\prime}$ are concave downwards.

The boundary $P_{0} Q P_{1} \bar{Q}$ of the primary region and the boundary $P_{0}^{\prime} Q^{\prime} P_{1}^{\prime} \bar{Q}^{\prime}$ of its transform under $T_{w}(1)$ are in one-to-one correspondence with $J^{(1)}>0$ holding in the interior of the primary region. It follows ${ }^{16}$ that the interiors of the two regions are in one-to-one correspondence to assign a unique state to each point of the region $P_{0}^{\prime} Q^{\prime} P_{1}^{\prime} \bar{Q}^{\prime}$ in Figure $3 \mathrm{~b}$.

5. The second initial value problem. To extend our knowledge of the states of the gas we draw propagation lines from the points of the arc $Q^{\prime} P_{1}^{\prime}$. These propagation lines are tangent to $r$-curves on $Q^{\prime} P_{1}^{\prime}$ and according to Lemma 8 do not intersect on the convex side of $Q^{\prime} P_{1}^{\prime}$.

The equation of the propagation line from $Q^{\prime}$ is

$$
x-\frac{2}{3}\left(2 r_{0}+s_{1}\right) t=w_{r}^{(1)}\left(r_{0}, s_{1}\right)=-1-2 \frac{r_{0}+s_{1}}{r_{0}-s_{1}},
$$

16 A beginning in this direction has been made by Rand, loc. cit.,

${ }^{16}$ See, for example, G. A. Bliss, Fundamental existence theorems vol. III, Amer. Math. Soc. Colloquium Publications, reprinted 1934, p. 42. 
and the equation of the shock line from $A$ is, from (13), since $r_{2}+s_{2}=0$,

$$
x-\frac{\left(r_{0}+s_{1}\right)\left(r_{0}-s_{1}\right)^{3}}{\left(r_{0}-s_{1}\right)^{3}-\left(r_{2}-s_{2}\right)^{3}} t=1 .
$$

The two lines intersect in a point $Q^{\prime \prime}$ with coordinates

$x^{\prime \prime}=1+\frac{12 r_{0}}{\lambda\left(r_{0}, s_{1}\right)}\left(r_{0}+s_{1}\right)\left(r_{0}-s_{1}\right)^{2}, \quad t^{\prime \prime}=\frac{12 r_{0}}{\lambda\left(r_{0}, s_{1}\right)} \frac{\left(r_{0}-s_{1}\right)^{3}-\left(r_{2}-s_{2}\right)^{3}}{r_{0}-s_{1}}$,

where

$$
\lambda(r, s)=(r-s)^{4}-2(2 r+s)\left(r_{2}-s_{2}\right)^{3} .
$$

Referring to Lemmas 1 and 2 a solution $w^{(2)}$ of $\left(16^{\prime}\right)$ transforms $Q P_{1}$ into an $s$-curve propagated from $Q^{\prime} P_{1}^{\prime}$ and containing $Q^{\prime \prime}$ if

$$
w_{r}^{(2)}\left(r, s_{1}\right)=w_{r}^{(1)}\left(r, s_{1}\right), \quad r_{1} \leqq r \leqq r_{0}, \quad w_{c}^{(2)}\left(r_{0}, s_{1}\right)=x^{\prime \prime}-\frac{2}{3}\left(r_{0}+2 s_{1}\right) t^{\prime \prime},
$$

the latter condition determining $w_{q}^{(2)}$ uniquely on $Q P_{1}$.

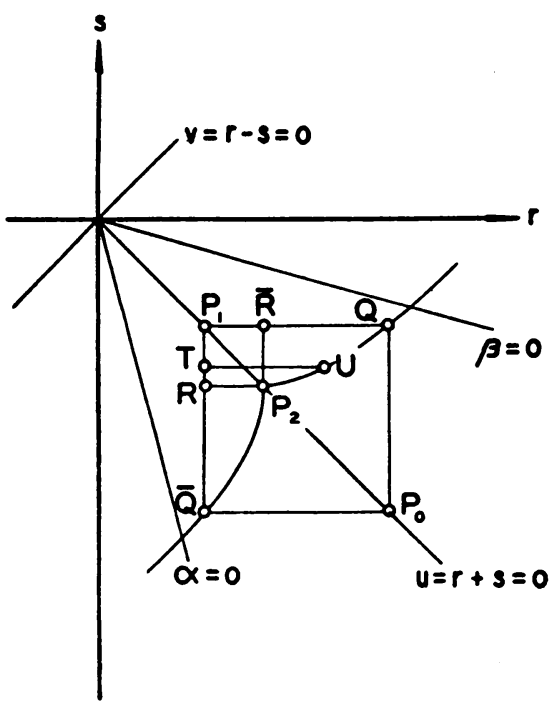

Fig. 4a.

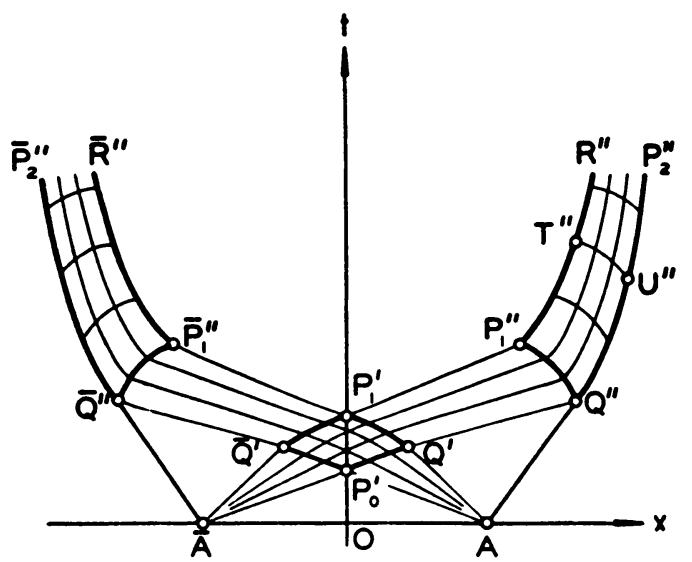

Fig. $4 b$.

Let the arc $Q^{\prime \prime} P_{2}^{\prime \prime}$ in Figure $4 \mathrm{~b}$ indicate the prolongation of the shock line $A Q^{\prime \prime}$. On the right of $Q^{\prime \prime} P_{2}^{\prime \prime}$ the state of the gas is $P_{2}\left(r_{2}, s_{2}\right)$ and the states immediately on the left of $Q^{\prime \prime} P_{2}^{\prime \prime}$ are represented by points $(r, s)$ on the compatibility curve (21). Thus $Q^{\prime \prime} P_{2}^{\prime \prime}$ is the transform by $T_{w(2)}$ of the compatibility curve $Q P_{2}$.

On the one hand the slope of $Q^{\prime \prime} P_{2}^{\prime \prime}$ is

$$
\frac{d t}{d x}=\frac{t_{r} r^{\prime}+t_{s}}{x_{r} r^{\prime}+x_{s}}=\frac{3}{2} \frac{t_{r} r^{\prime}+t_{s}}{(r+2 s) t_{r} r^{\prime}+(2 r+s) t_{s}},
$$

where $r=r(s)$ is defined implicitly by (21) and its derivative $r^{\prime}$ is

$$
r^{\prime}=\frac{8(r-s)^{5}-20\left(r_{2}-s_{2}\right)^{3}(r+2 s)^{2}-3\left(r_{2}-s_{2}\right)^{5}}{8(r-s)^{5}-20\left(r_{2}-s_{2}\right)^{3}(2 r+s)^{2}-3\left(r_{2}-s_{2}\right)^{5}} .
$$


On the other hand, from (13)

$$
\frac{d t}{d x}=\frac{(r-s)^{3}-\left(r_{2}-s_{2}\right)^{3}}{(r+s)(r-s)^{3}}
$$

and a comparison of the two results yields the condition

$$
\frac{t_{s}}{t_{r}}=\frac{\mu(r, s)}{\lambda(r, s)} r^{\prime}=\delta, \quad \mu(r, s)=(r-s)^{4}+2(r+2 s)\left(r_{2}-s_{2}\right)^{3},
$$

along $Q P_{2}$, or

$$
\delta w_{r r}^{(2)}-2(1+\delta) w_{r s}^{(2)}+w_{s s}^{(2)}=0 .
$$

The Second Initial Value Problem. To construct a solution $w^{(2)}$ of $\left(16^{\prime}\right)$ meeting the conditions (30) on the side $Q P_{1}$ of the primary region and the condition (32') along the arc $Q P_{2}$ of the compatibility curve.

From (24) the first condition in (30) is met by taking

$$
R=r_{0}^{2}-r^{2}, \quad S\left(s_{1}\right)=s_{1}^{2}-s_{0}^{2},
$$

in (17) and, from (28), the second condition determines

$$
S^{\prime}\left(s_{1}\right)=2 s_{1}-4 r_{0} \frac{\mu\left(r_{0}, s_{1}\right)}{\lambda\left(r_{0}, s_{1}\right)} .
$$

The parametric equations of the arc $Q^{\prime \prime} P_{1}^{\prime \prime}$ are obtained by placing $s=s_{1}$ in $\left(18^{\prime}\right)$ and substituting for $R, S\left(s_{1}\right), S^{\prime}\left(s_{1}\right)$ from (33), (34). In particular it is readily verified that $x\left(P_{1}^{\prime \prime}\right)>1$.

Taking condition $\left(32^{\prime}\right)$ in the form (32), and substituting for $t_{r}, t_{s}$ from (19) with $R=r_{0}^{2}-r^{2}$ it will be found that this condition becomes ${ }^{17}$

$$
S^{\prime \prime}+2 \frac{2+\delta}{r-s} S^{\prime}+6 \frac{1+\delta}{(r-s)^{2}} S=\frac{(r-s)^{2}}{3}\left(t_{s}^{(1)}-\delta t_{r}^{(1)}\right),
$$

where $\delta, t_{r}^{(1)}, t_{s}^{(1)}$ are the rational functions of $r, s$ defined in (32), (26), and $r$ is the algebraic function of $s$ defined in (21) with $r_{2}+s_{2}=0$. Thus to obtain the solution $w^{(2)}$ of the second initial value problem we set $R=r_{0}^{2}-r^{2}$ in (17) and choose $S$ to be the solution of the ordinary differential equation of second order (35), subject to the initial conditions in (33), (34).

Lemma 9. The value of $\delta$ at a point $P$ of the compatibility curve $Q P_{2}$ tends to $+\infty$ as $P$ tends to $P_{2}$. More precisely $\delta$ is a positive regular analytic function of the parameter $v$ on $Q P_{2}$, except at $v=v_{2}$, where it has a pole of the third order and a Laurent expansion of the form

$$
\delta=v_{2}^{3}\left(v-v_{2}\right)^{-3}(1+\cdots) .
$$

To prove $\delta>0$ we have

$$
\lambda=v\left(v^{3}-v_{2}^{3}\right)-3 u v_{2}^{3}, \quad \mu=v\left(v^{3}-v_{2}^{3}\right)+3 u v_{2}^{3},
$$

${ }^{17}$ The form of the second member in (35) is due to Rand. 
and the equation of the compatibility curve $Q P_{2}$

$$
15 v_{2}^{3} u^{2} v^{3}=\left(v^{5}-v_{2}^{5}\right)\left(v^{3}-v_{2}^{3}\right) \text {. }
$$

It is obvious that $\mu>0$ for $v>v_{2}$, and $\lambda>0$ follows from $\lambda \mu=v^{2}\left(v^{3}-v_{2}^{3}\right)^{2}-9 u^{2} v_{2}^{6}>0$ for $v>v_{2}$. To establish this inequality, one multiplies the equation of the compatibility curve by $3 v_{2}^{3}$ and observes that $3 v_{2}^{3}\left(v^{5}-v_{2}^{5}\right)<5 v^{5}\left(v^{3}-v_{2}^{3}\right)$ holds for $v>v_{2}$. Now $r^{\prime}>0$ by Lemma 6 and therefore $\delta>0$ for $v>v_{2}$ by (32).

From (20) one has $u=r+s=v-v_{2}+2 \kappa\left(v-v_{2}\right)^{3}+\cdots$, so that

$\lambda=6 v_{2}^{2}\left(v-v_{2}\right)^{2}+\cdots, \quad \mu=6 v_{2}^{3}\left(v-v_{2}\right)+\cdots, \quad r^{\prime}=v_{2}^{2}\left(v-v_{2}\right)^{-2}(1+\cdots)$,

hold along $Q P_{2}$, and the Laurent expansion for $\delta$ then follows from (32).

It is apparent from (20) and Lemma 9 that the coefficients of the differential equation (35) present a singular point at $s=s_{2}$.

LEMMA 10. The introduction of $v$ as independent variable in the differential equation (35) leads to a differential equation for $V=S(s(v))$ in which the coefficients are regular analytic functions of $v$ for $v \geqq v_{2}$.

Retaining the prime to denote differentiation with respect to $s$ and indicating differentiation with respect to $v$ by a dot, so that $S^{\prime}=\dot{V} / s, S^{\prime \prime}=(s \ddot{V}-\dot{V} s) / s^{3}$, the differential equation (35) becomes

$$
\ddot{V}+(2(2+\delta) s / v-\ddot{s} / s) \dot{V}+6 \dot{s}^{2} v^{-2}(1+\delta) V=\frac{v^{2} s^{2}}{3}\left(t_{s}^{(1)}-\delta t_{r}^{(1)}\right),
$$

in which the coefficients are regular analytic functions of $v$ for $v>v_{2}$ by Lemmas 6 and 9. Moreover if the coefficients are expanded in powers of $v-v_{2}$ using (20) and (36), it will be found that they are also regular about $v_{2}$.

Lemma 11. Provided $\rho_{0}-\rho_{2}>0$ is sufficiently small, $S$ and $S^{\prime}$ are negative for $s_{2} \leqq s \leqq s_{1}$ with $S$ tending to a finite limit and $S^{\prime}$ to $-\infty$ as $s$ approaches $s_{2}$.

Since the coefficients in (38) are regular at $v=v_{2}$, the solution determined by $V\left(v_{0}\right)=V_{0}, \dot{V}\left(v_{0}\right)=\dot{V}_{0}$ may be expanded ${ }^{18}$ in a power series in $v-v_{2}, v_{0}-v_{2}, V_{0}, \dot{V}_{0}$ provided the absolute values of these quantities are sufficiently small.

Taking $v_{0}$ for the value of $v$ corresponding to point $Q$ on the compatibility curve, $v_{0}-v_{2}$ can be made arbitrarily small by taking $\rho_{0}-\rho_{2}$ sufficiently small, with the coordinates of $Q$ given by

$$
r_{0}=r_{2}+v_{0}-v_{2}+\kappa\left(v_{0}-v_{2}\right)^{3}+\cdots, \quad s_{1}=s_{2}+\kappa\left(v_{0}-v_{2}\right)^{3}+\cdots .
$$

The initial conditions for $S$ in (33), (34) lead to the initial conditions

$$
V\left(v_{0}\right)=s_{1}^{2}-r_{0}^{2}, \quad \dot{V}\left(v_{0}\right)=2 s\left(v_{0}\right)\left[s_{1}-2 r_{0} \frac{\mu\left(r_{0}, s_{1}\right)}{\lambda\left(r_{0}, s_{1}\right)}\right]
$$

for $V$. From (37), (39) we obtain the expansions

$$
V\left(v_{0}\right)=-v_{2}\left(v_{0}-v_{2}\right)-\left(v_{0}-v_{2}\right)^{2}+\cdots, \quad \dot{V}\left(v_{0}\right)=-2\left(v_{0}-v_{2}\right)+\cdots,
$$

${ }^{18} \mathrm{~J}$. Horn, Gewöhnlichs Differentialgleichungen beliebiger Ordnung, Sammlung Schubert, vol. 50, Leipzig, 1905, pp. 27-28. 
valid for sufficiently small $\left|v_{0}-v_{2}\right|$. It follows from (38) that the expansion of $\ddot{V}\left(v_{0}\right)$ in powers of $v_{0}-v_{2}$ begins with a term of at least first degree in $v_{0}-v_{2}$.

When the expansions (41) are substituted in the expansion of the solution $V$ in powers of $v-v_{2}, v_{0}-v_{2}, V_{0}, \dot{V}_{0}$ it appears that $V$ may be expanded in powers of $v-v_{2}$, $v_{0}-v_{2}$ provided $\left|v-v_{2}\right|,\left|v_{0}-v_{2}\right|$ are sufficiently small. To obtain the linear and quadratic terms of this expansion, we substitute from (41) in Taylor's series

$$
V=V\left(v_{0}\right)+\dot{V}\left(v_{0}\right)\left(v-v_{0}\right)+\frac{\ddot{V}\left(v_{0}\right)}{2}\left(v-v_{0}\right)^{2}+\cdots
$$

to obtain

$$
V=-v_{2}\left(v_{0}-v_{2}\right)-2\left(v_{0}-v_{2}\right)\left(v-v_{2}\right)+\left(v_{0}-v_{2}\right)^{2}+\cdots,
$$

the third term in Taylor's series being neglected since $\ddot{V}\left(v_{0}\right)$ contains the factor $v_{0}-v_{2}$.

It follows that both $V, \dot{V}$ are negative for $v_{2} \leqq v \leqq v_{0}$ for sufficiently small $v_{0}-v_{2}>0$. It is clear that $S$ tends to a finite negative limit as $s$ tends to $s_{2}$ and, since $s$ is positive and tends to zero as $v$ tends to $v_{2}$, one concludes that $S^{\prime}$ tends to $-\infty$ as $s$ tends to $s_{2}$, provided, of course, that $\rho_{0}-\rho_{2}$ is sufficiently small.

The subregion $P_{2} Q P_{1} R P_{2}$ in Figure 4 a of the primary region is termed the secondary region.

Lemma 12. The partial derivatives $t_{r}^{(2)}, t_{s}^{(2)}$ and the Jacobian $J^{(2)}=-2 G t_{r}^{(2)} t_{s}^{(2)}$ of $T_{w(2)}$ are negative in the secondary region for sufficiently small $\rho_{0}-\rho_{2}>0$.

We take $R, S$ in (19) as determined by the second initial value problem and find

$$
t_{r}^{(2)}=\frac{1}{2} t_{r}^{(1)}+\frac{3 S^{\prime}}{(r-s)^{3}}+\frac{9 S}{(r-s)^{4}}, t_{c}^{(2)}=\frac{1}{2} t_{s}^{(1)}-\frac{3}{2} \frac{S^{\prime \prime}}{(r-s)^{2}}-\frac{6 S^{\prime}}{(r-s)^{3}}-\frac{9 S}{(r-s)^{4}},
$$

from which $t_{r}^{(2)}<0$ follows from (27) and Lemma 11 for sufficiently small $\rho_{0}-\rho_{2}>0$.

To prove $t_{s}^{(2)}<0$ we have

$$
S^{\prime \prime}+2 \frac{2+\bar{\delta}}{\bar{r}-s} S^{\prime}+6 \frac{1+\bar{\delta}}{(\bar{r}-s)^{2}} S=\frac{(\bar{r}-s)^{2}}{3}\left(\bar{t}_{s}^{(1)}-\bar{\delta}_{t_{r}}^{(1)}\right),
$$

where $\bar{r}=r(s)$ is the function of $s$ defined in Lemma 6 and $\bar{\delta}=\delta(\bar{r}, s), \bar{t}_{r}^{(1)}=t_{r}^{(1)}(\bar{r}, s)$, $\bar{t}_{s}^{(1)}=t_{s}^{(1)}(\bar{r}, s)$. When $S^{\prime \prime}$ is eliminated from $t_{s}^{(2)}$ it is found that $t_{s}^{(2)}=A S^{\prime}+B S+C$, where

$$
\begin{aligned}
A & =\frac{6}{(\bar{r}-s)(r-s)^{2}}\left[1+\frac{\bar{\delta}}{2}-\frac{\bar{r}-s}{r-s}\right]>6(\bar{r}-s)^{-1}(r-s)^{-2}\left(\frac{\bar{\delta}}{2}-\frac{r_{0}}{r_{1}}\right), \\
B & =\frac{9}{(r-s)^{2}(\bar{r}-s)^{2}}\left[1+\bar{\delta}-\left(\frac{\bar{r}-s}{r-s}\right)^{2}\right]>9(r-s)^{-2}(\bar{r}-s)^{-2}\left(\bar{\delta}-\frac{r_{0}^{2}}{r_{1}^{2}}\right), \\
C & =\frac{1}{2} t_{s}^{(1)}+\frac{1}{2}\left(\frac{\bar{r}-s}{r-s}\right)^{2}\left(\bar{\delta} \bar{t}_{r}^{(1)}-\bar{t}_{s}^{(1)}\right)<\frac{1}{2}\left(\bar{\delta}_{r}^{(1)}+\bar{t}_{s}^{(1)}\right),
\end{aligned}
$$

in view of the inequalities

$$
2 r_{1}=r_{1}-s_{1}<r-s \leqq \bar{r}-s<r_{0}-s_{0}=2 r_{0},
$$


valid in the secondary region. $t_{r}^{(1)}$ has a negative upper bound and $t_{s}^{(1)}$ a positive lower bound in the secondary region independent of $\rho_{0}$. Moreover $r_{0}, r_{1}$ tend to $r_{2}$ as $\rho_{0}$ approaches $\rho_{2}$. In view of Lemma 9 we have $A>0, B>0, C<0$, and therefore $t_{s}^{(2)}<0$ by Lemma 11 for sufficiently small $\rho_{0}-\rho_{2}$.

We shall now investigate the mapping by $T_{w^{(2)}}$ of the secondary region upon the $(x, t)$-plane. Taking $R, S$ in $\left(18^{\prime}\right)$ as determined above in the solution of the second initial value problem, $T_{w^{(2)}}$ is

$$
\begin{aligned}
x^{(2)} & =\frac{(r+s)\left(3 r_{0}^{2}-r^{2}\right)-4 r s^{2}}{(r-s)^{3}}-3 \frac{r+s}{(r-s)^{3}} S-\frac{2 r+s}{(r-s)^{2}} S^{\prime}, \\
t^{(2)} & =3 \frac{r_{0}^{2}-r s}{(r-s)^{3}}-\frac{3 S}{(r-s)^{3}}-\frac{3}{2} \frac{S^{\prime}}{(r-s)^{2}} .
\end{aligned}
$$

From Lemma 11 it follows (at least for $\rho_{0}-\rho_{2}$ sufficiently small) that $x^{(2)}, t^{(2)}$ become infinite as the point $(r, s)$ of the secondary region approaches the side $R P_{2}$. We shall accordingly consider first the mapping by $T_{w(2)}$ of the subregion $U Q P_{1} T U$, the line $T U$ being parallel to $R P_{2}$.

Sides $P_{1} Q, T U$ transform into $s$-curves $P_{1}^{\prime \prime} Q^{\prime \prime}, T^{\prime \prime} U^{\prime \prime}$. From Lemma $12 t$ decreases and from (5) $x$ increases as $r$ increases along $P_{1} Q, T U$. We conclude from Lemma 7 that $P_{1}^{\prime \prime} Q^{\prime \prime}, T^{\prime \prime} U^{\prime \prime}$ are concave downward as shown in Figure $4 \mathrm{~b}$.

Side $P_{1} T$ transforms into an $r$-curve $P_{1}^{\prime} T^{\prime \prime}$ which is concave upwards.

Arc $Q U$ of the compatibility curve transforms into the shock curve $Q^{\prime \prime} U^{\prime \prime}$. Along $Q U x$ and $t$ are monotonic decreasing functions of $v$, as is the slope $d t / d x$ of $Q^{\prime \prime} U^{\prime \prime}$, for, from (31)

$$
\frac{d}{d v}\left(\frac{d t}{d x}\right)=-\frac{\lambda \dot{r}+\mu \dot{s}}{(r+s)^{2}(r-s)^{4}}
$$

inasmuch as $\lambda>0, \mu>0$ hold on $Q U$. The shock curve is accordingly concave upwards to imply that the velocity of propagation of the shock decreases as $t$ increases.

Finally we let $U$ approach $P_{2}$ along $Q P_{2}$. The $s$-curve $T^{\prime \prime} U^{\prime \prime}$ recedes to infinity in the $(x, t)$-plane and the secondary region, exclusive of side $R P_{2}$, is accordingly mapped in (1-1) fashion by $T_{w(2)}$ upon a region indicated by $P_{2}^{\prime \prime} Q^{\prime \prime} P_{1}^{\prime} R^{\prime \prime}$ in the $(x, t)$-plane to determine the states of the gas in this region.

The slope of the $r$-curve $P_{1}^{\prime \prime} T^{\prime \prime}$ at $T^{\prime \prime}$ tends to $1 / \alpha\left(r_{1}, s_{2}\right)$ as $T^{\prime \prime}$ recedes to infinity. The slope of the shock curve at $U^{\prime \prime}$ is, from (11),

$$
\frac{d t}{d x}=\sqrt{\frac{\rho_{2}}{\rho} \frac{\rho-\rho_{2}}{p-p_{2}}}
$$

where $\rho$ denotes the density for the state $U$ and $p=p(\rho)$. As $U \rightarrow P_{2}$ we have $\rho \rightarrow \rho_{2}$, which implies $d t / d x \rightarrow 1 / G_{2}$. This means that the velocity of propagation of the shock tends toward the local velocity of sound in the exterior body of gas through which the shock travels as it recedes to infinity.

The determination of the states of the gas in the region $\bar{P}_{2}^{\prime \prime} \bar{Q}^{\prime \prime} \bar{P}_{1}^{\prime \prime} \bar{R}^{\prime \prime}$ may now be left to symmetry considerations or Theorem 1 , 
6. The third initial value problem. We take up the problem of determining the states of the gas in the region of the $(x, t)$-plane lying above the curve $\bar{R}^{\prime \prime} \bar{P}_{1}^{\prime} P_{1}^{\prime} P_{1}^{\prime} R^{\prime \prime}$ in Figure $4 \mathrm{~b}$.

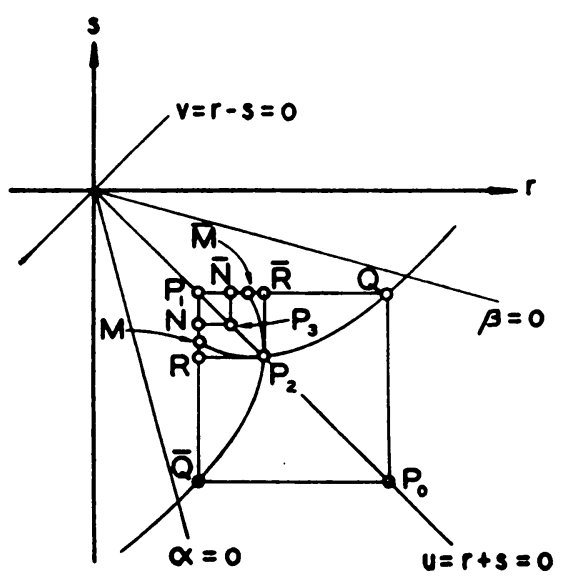

Fig. 5a.

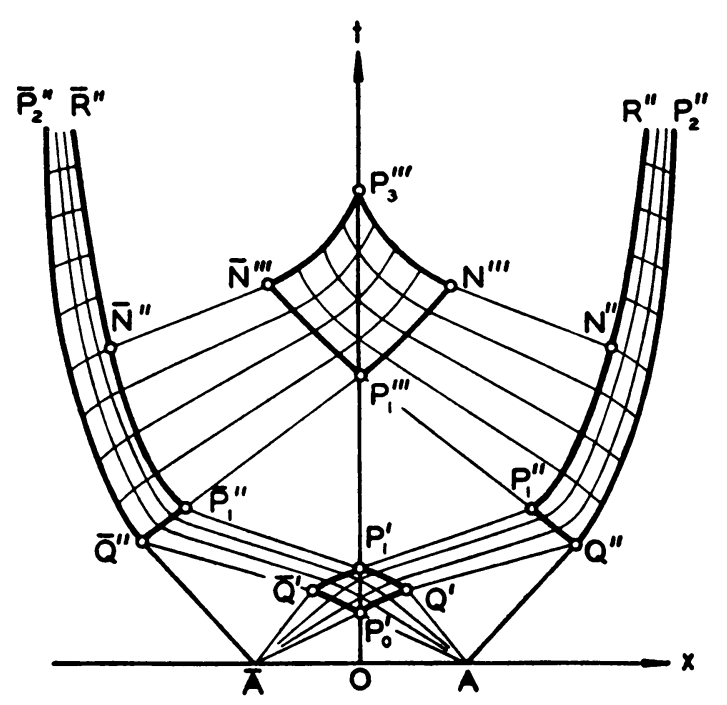

Fig. 5b.

Propagation lines drawn from $P_{1}^{\prime \prime} R^{\prime \prime}$ in Figure $5 \mathrm{~b}$ have slope $1 / \beta<0$ and, from Lemma 8 can intersect only on the concave side of $P_{1}^{\prime \prime} R^{\prime \prime}$. We shall prove that they do not meet in the region $x>0$ if $\rho_{0}-\rho_{2}$ is sufficiently small. Since $\phi$ is a monotonic decreasing function of $s$ by Lemma 7, it will be sufficient to prove that the $t$-intercept $T$ of a propagation line is a monotonic decreasing function of $s$.

In the equation of a propagation line $t=\beta^{-1} x+T$ we replace $x, t$ by the coordinates of a point on $P_{1}^{\prime \prime} R^{\prime \prime}$ obtained from $T_{v(2)}$ to obtain

$$
T=-\frac{w^{(2)}\left(r_{1}, s\right)}{\beta\left(r_{1}, s\right)}=\frac{\left(r_{1}-s\right) S^{\prime}+S+r_{1}^{2}-r_{0}^{2}}{\beta\left(r_{1}, s\right)\left(r_{1}-s\right)^{2}},
$$

from which

$$
\frac{d T}{d s}=\frac{\beta\left(r_{1}, s\right)\left(r_{1}-s\right)^{2} S^{\prime \prime}+4 s\left(r_{1}-s\right) S^{\prime}+4 s S+4 s\left(r_{1}^{2}-r_{0}^{2}\right)}{\beta^{2}\left(r_{1}, s\right)\left(r_{1}-s\right)^{3}} .
$$

After $S^{\prime \prime}$ is eliminated by (35) it will be found that $d T / d s<0$ holds for sufficiently small $\rho_{0}-\rho_{2}$. The principle of the argument is essentially the same as the one employed to prove that $t_{s}^{(2)}<0$ in Lemma 12 and is omitted.

From symmetry considerations propagation lines drawn from $\bar{P}_{1}^{\prime \prime} \bar{R}^{\prime \prime}$ do not intersect in the region $x<0$. Propagation lines drawn from $P_{1}^{\prime \prime} R^{\prime \prime}$ and $\bar{P}_{1}^{\prime \prime} \bar{R}^{\prime \prime}$ symmetrically placed with respect to the $t$-axis intersect upon it and, excepting the two drawn from $P_{1}^{\prime \prime}, \bar{P}_{1}^{\prime \prime}$, assign different states to their points of intersection. This is avoided in Figure $5 \mathrm{~b}$ by terminating the propagation lines on arcs $P_{1}^{\prime \prime} N^{\prime \prime \prime}, P_{1}^{\prime \prime} \bar{N}^{\prime \prime}$, the coordinates of $P_{1}^{\prime \prime \prime}$ being $x=0, t=-w_{s}^{(2)}\left(r_{1}, s_{1}\right) / \beta\left(r_{1}, s_{1}\right)$. 
By Lemma 1 an $r$-curve, the transform of $P_{1} R$ by $T_{w(z)}$ will be propagated from $P_{1}^{\prime \prime} R^{\prime \prime}$ provided

$$
w_{s}^{(3)}\left(r_{1}, s\right)=w_{s}^{(2)}\left(r_{1}, s\right) \text { for } s_{2} \leqq s \leqq s_{1},
$$

and by Lemma 2 will contain $P_{1}^{\prime}{ }^{\prime}$, in case

$$
w_{r}^{(3)}\left(r_{1}, s_{1}\right)=\frac{\alpha\left(r_{1}, s_{1}\right)}{\beta\left(r_{1}, s_{1}\right)} w_{*}^{(2)}\left(r_{1}, s_{1}\right)=-w_{*}^{(2)}\left(r_{1}, s_{1}\right) .
$$

At points symmetric to the $t$-axis states have the same density and opposite velocities. From the corollary to Theorem 1 this will be the case in the region above $\bar{N}^{\prime \prime \prime} P_{1}^{\prime}{ }^{\prime} N^{\prime \prime \prime}$ if this region is the transform by $T_{w}$ of a region in the state plane symmetric to the line $r+s=0$, provided $w(r, s)=w(-s,-r)$ holds in this region.

The Third Initial Value Problem. To construct a solution $w^{(3)}$ of $\left(16^{\prime}\right)$ meeting the symmetry condition $w^{(3)}(r, s)=w^{(3)}(-s,-r)$ and the initial conditions (42), (43) on the side $P_{1} R$ of the secondary region.

The solution of this initial value problem

$$
w^{(s)}(r, s)=-\frac{S(-r)+S(s)}{r-s},
$$

is obtained by setting $R=-S(-r)$ in (17), where $S(s)$ is the function entering in the solution of the second initial value problem.

The symmetry condition is obviously fulfilled. From (33) we find $w^{(3)}\left(r_{1}, s\right)$ $=w^{(2)}\left(r_{1}, s\right)$ and (42) follows by differentiation. Condition (43) is likewise a consequence of (33).

The subregion $P_{1} R P_{2} \bar{R} P_{1}$ of the secondary region in Figure $5 \mathrm{a}$ is termed the tertiary region.

The mapping by $T_{w(3)}$ of the tertiary region upon the $(x, t)$-plane is not (1-1). If $R$ is replaced by $-S(-r)$ in (19) one obtains

$$
t_{r}^{(3)}=\frac{3}{2} \frac{(r-s)^{2} S^{\prime \prime}(-r)+2(r-s)\left[2 S^{\prime}(-r)+S^{\prime}(s)\right]+6[S(-r)+S(s)]}{(r-s)^{4}} .
$$

In particular on $r=-s$ (along $\left.P_{1} P_{2}\right)$

$$
t_{r}^{(3)}=\frac{3}{8 s^{4}}\left[s^{2} S^{\prime \prime}(s)-3 s S^{\prime}(s)+3 S(s)\right] .
$$

For $\rho_{0}-\rho_{2}$ sufficiently small $t_{r}^{(3)}$ is positive along $P_{1} P_{2}$ in view of (27), (35) and Lemmas 9 and 11. On the other hand, if we fix $r$ in (45) and allow $s$ to approach $s_{2}$ it appears that $t_{r}^{(3)}$ eventually becomes negative because of the behavior of $S, S^{\prime}$ as $s$ tends to $s_{2}$. Hence there exists a subregion $P_{1} P_{2} M P_{1}$ of the tertiary region within which $t_{r}^{(3)}$ is nositive, except along $M P_{2}$ where $t_{r}^{(3)}=0$.

Differentiating equations (5) partially with respect to $r$ and $s$ and eliminating $x_{r s}$, we find that $t$ satisfies the partial differential equation $(\alpha-\beta) \iota_{r s}=\beta_{s} \iota_{r}-\alpha_{r} l_{s}$ which reduces, in the adiabatic case, to

$$
(r-s) t_{r s}=\frac{1}{2} \frac{\gamma+1}{\gamma-1}\left(t_{r}-t_{s}\right)
$$



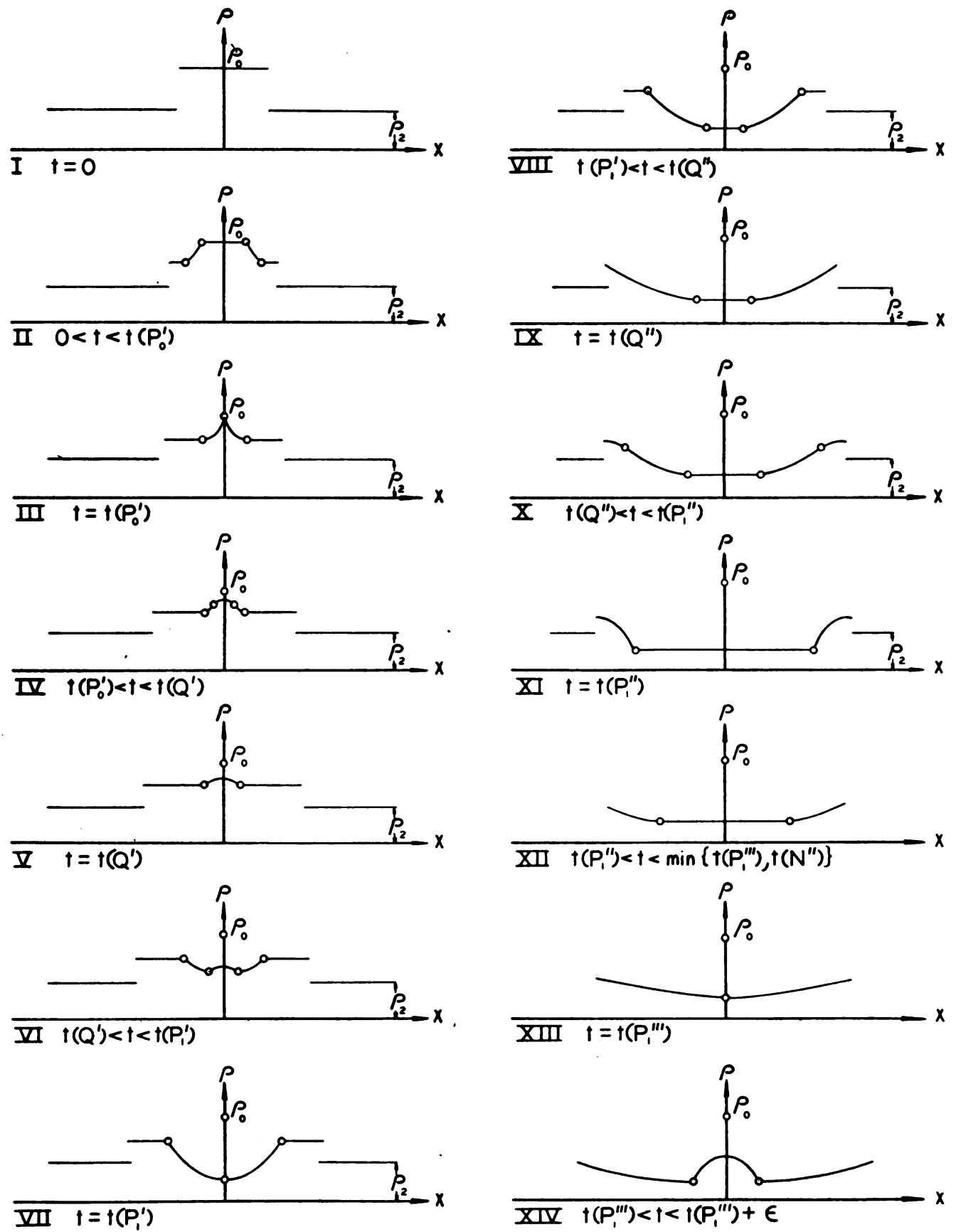

Plate 1. Variation of density $\rho$ with distance $x$ for fixed time $t$. 

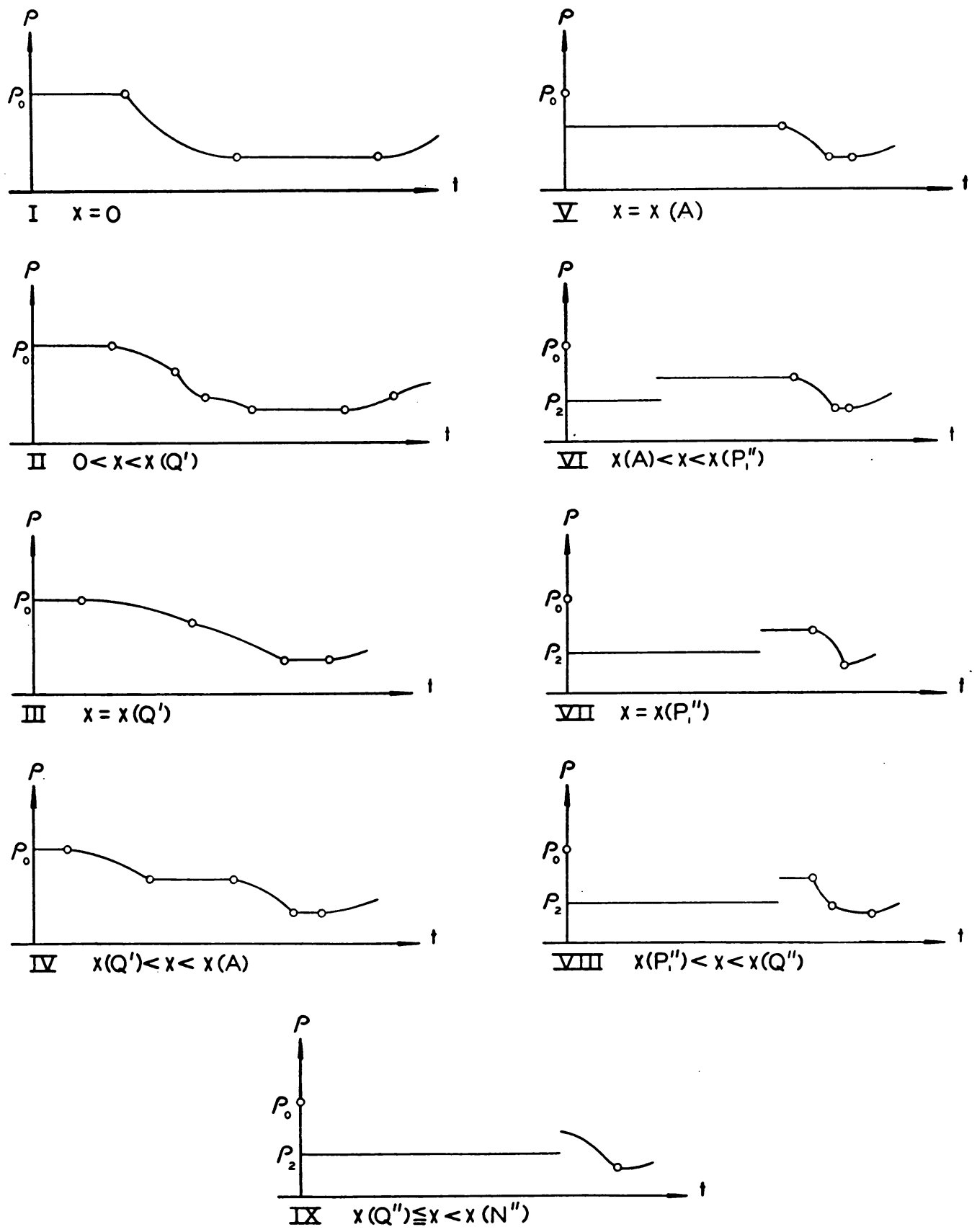

Plate 2. Variation of density $\rho$ with time $t$ for fixed distance $x$. 
It follows that in a region in which $t_{r}$ is positive and $t_{\mathrm{s}}$ is negative, $t_{r}\left(t_{\mathrm{s}}\right)$ is a monotonic increasing function of $s(r)$ for fixed $r(s)$.

From $t^{(3)}(r, s)=t^{(3)}(-s,-r)$ we have $t_{s}^{(3)}(r, s)=-t_{r}^{(3)}(-s,-r)$ and thus $t_{s}^{(3)}=-t_{r}^{(3)}$ along $P_{1} P_{2}$. It follows that $t_{3}^{(3)}$ is negative along $P_{1} P_{2}$, and consequently is negative in a suitably restricted region containing $P_{1} P_{2}$. When we procede from $P_{1} P_{2}$ to the left along a line $s=$ const., $t_{s}^{(3)}$ decreases and accordingly is negative everywhere in $P_{1} P_{2} M P_{1}$.

Along $M P_{2} \partial t_{r}^{(3)} / \partial s$ is positive and $J_{r}^{(3)}=-2 G t_{r}^{(3)} t_{s}^{(3)}$ changes sign as $(r, s)$ crosses $M P_{2}$. Thus the mapping of the tertiary region cannot be (1-1).

It is clear that $t_{s}^{(3)}$ vanishes along an arc $\bar{M} P_{2}$, the reflection of $M P_{2}$ in $P_{1} P_{2}$. Within the region $P_{1} M P_{2} \bar{M} P_{1}$ we have $J^{(3)}>0$. The application of $T_{w}(3)$ to the tertiary region to obtain further information about the states of the gas must be restricted to a square $P_{1} N P_{3} \bar{N}$ within the region $P_{1} M P_{2} \bar{M} P_{1}$ with sides on $P_{1} M, P_{1} \bar{M}$. Such a square is carried by $T_{w(3)}$ into the region $P_{1}^{\prime \prime} N^{\prime \prime \prime} P_{3}^{\prime \prime} \bar{N}^{\prime \prime \prime}$ of Figure $5 \mathrm{~b}$, within which the states of the gas may be regarded as known.

The prolongation of the solution into the rest of the $(x, t)$-plane still awaits solution and it should be noted here that further extension of the solution may modify the states assigned above to the region $R^{\prime \prime} P_{1}^{\prime \prime} Q^{\prime \prime} P_{2}^{\prime \prime}$ and its reflection in the $t$-axis.

7. Graphical presentation of variation of density. Plates 1 and 2 portray the variation of density (and thus the pressure) of a monatomic gas for sufficiently small $\rho_{0}-\rho_{2}$ in so far as our analysis permits. They were obtained by comparing Figures 5a and $5 \mathrm{~b}$ with the aid of (2). No attempt was made to indicate quantitative changes in the density, or to determine the curvature of the curved portions of the graphs. The small circles indicate points at which $\rho_{x}$ and $\rho_{t}$ undergo jumps. Figures VIII, IX of Plate 2 are based on the conjecture that $x\left(Q^{\prime \prime}\right)<x\left(N^{\prime \prime}\right)$.

The coordinates of various points in Figure $5 b$ have been computed from formulas found in the text and are given below. Once $\rho_{0}, \rho_{2}$ are given, $r_{0}, r_{2}$ are determined from (2) and $s_{1}$ may be determined graphically as the $s$-coordinate of $Q$ in Figure 5a.

$$
\begin{aligned}
& P_{0}^{\prime}: \quad x=0, \quad t=3 / 2 r_{0},=\frac{6 r_{0}}{\left(r_{0}-s_{1}\right)^{2}}, \quad t=\frac{\left.s_{1}\right)\left(5 r_{0}+s_{1}\right)}{\left(r_{0}-s_{1}\right)^{2}}, \\
& Q^{\prime}: \quad x=\frac{3}{4} \frac{r_{0}^{2}+r_{1}^{2}}{r_{1}^{3}}, \\
& P_{1}^{\prime}: \quad x=0, \quad t=\frac{2 r_{0}+s_{1}}{Q^{\prime \prime}:} \quad x=x\left(Q^{\prime}\right)+4 r_{0} \frac{\mu\left(r_{0}, s_{1}\right)}{\lambda\left(r_{0}-s_{1}\right)^{2}}, \quad t=t\left(Q^{\prime}\right)+\frac{6 r_{0}}{\left(r_{0}-s_{1}\right)^{2}} \frac{\mu\left(r_{0}, s_{1}\right)}{\lambda\left(r_{0}, s_{1}\right)}, \\
& P_{1}^{\prime \prime}: \quad x=\frac{1}{2}+\frac{r_{0}}{r_{1}} \frac{\mu\left(r_{0}, s_{1}\right)}{\lambda\left(r_{0}, s_{1}\right)}, \quad t=t\left(P_{1}^{\prime}\right)+\frac{3}{2} \frac{r_{0}}{r_{1}^{2}} \frac{\mu\left(r_{0}, s_{1}\right)}{\lambda\left(r_{0}, s_{1}\right)} \\
& P_{1}^{\prime \prime \prime}: x=0, \quad t=t\left(P_{1}^{\prime}\right)+3 \frac{r_{0}}{r_{1}} \frac{\mu\left(r_{0}, s_{1}\right)}{\lambda\left(r_{0}, s_{1}\right)} .
\end{aligned}
$$

